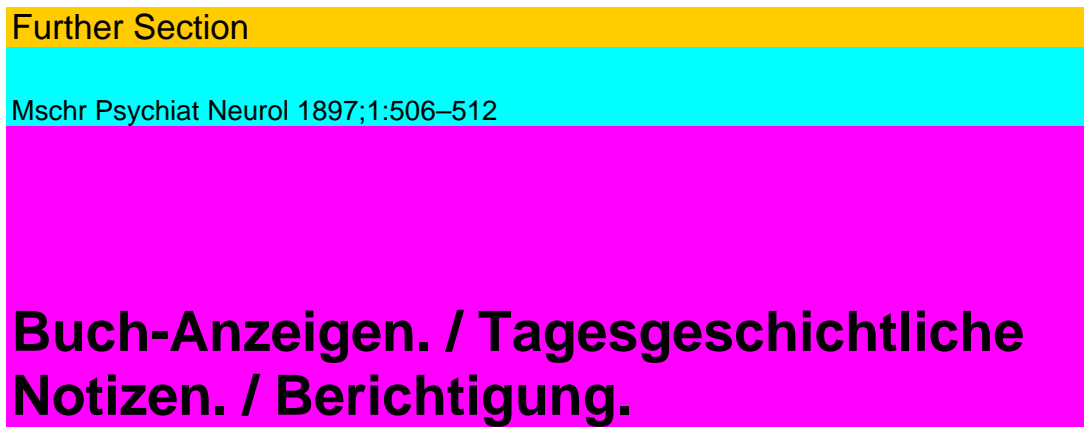

Die Irren-Anstalt Herzberge der Stadt Berlin in Lichtenberg mit Bemerkungen über Bau und Einrichtungen von Anstalten für Geisteskranke. Von C. Moeli. Berlin 1896. Otto Enslin.

Die psychiatrische Litteratur besitzt nicht viele Abhandlungen über Einrichtung von Irrenanstalten, die über das Mass einer einfachen Beschreibung hinausgehend allgemeine Gesichtspunkte betonen. Anders das vorliegende Büchlein namentlich in seinem letzten Abschnitt.

Beim ersten Theil wird der Leser sich nicht dem Eindruck ver-schliessen können, dass- die Einrichtungen der Anstalt Herzberge bis in die

J) Medierna practica, 1889. Revista de Ciencias Medicas de Barcelona, Nos 21 u. 22, 1891.

a) La ceUule vol. 7 p. 101, 1891

3) Psyehology und cf. Waller, Science Progress, vol. 3, p. 186, May 1895.

B Arch. f. Anat. n. Physiol. 1859, p. 595.

») Du Bois Reymond's Arch. f. Physiol. 1877, p. 66.

*) Sherrington, Roy. Soc. Proc. vol. 60, p. 414

!) Ibid. 
Buchanzeigen. 
geringsten Einzelheiten auf das sorgfältigste unter Berücksichtigung' aller Eventualitäten vorbedacht und ausgeführt sind. Die elektrische Beleuchtung gewährt ausser völliger Eliminierung der Feuersgefahr - die Anwendung des Dreileitersystems machte eine Nothbeleuchtung überflüssig - durch Anbringung der Dunkelsteller die Möglichkeit einer leichten und schnellen Regulirung. Bemerk enswerth ist das Fehlen einer Schutzvorrichtung (Drahtnetz) um die Glühlampen, welches voll und ganz durch die Erfahrung gerechtfertigt ist, dass auch die erregtesten Kranken keine Zerstörungsversuche gemacht haben. Die Vertheilung der Baderäume ist sehr zweckmässig, namentlich im Pflegehaus, wo zwischen je 2 Sälen der Abtheilung für Bettlägerige ein Badezimmer mit Wascheinrichtung sich befindet. Bemerkens-werth ist der Luxus eines Schwimmbassins. Um den Charakter des Krankenhauses zu wahren, ist von einer Sicherung der Fenster im Pflegehause Abstand genommen, dagegen sind die verschiedensten Ventilationsvorrichtungen angebracht; es lässt sich dabei die Erwägung nicht unterdrücken, dass deren Bedienung nicht sehr einfach ist und andererseits keine Ventilation der durch die geöffneten unteren Fensterflügel gleichkommt. M. giebt dann seiner Ueberzeugung Ausdruck, dass in Baracken am besten die körperlicher Pflege bedürftigen Geisteskranken untergebracht sind.

Einige Worte widmet der Verf. auch der "Wärterfrage; er hat durch Gewährung besonderer Schlafrä'ume für das Personal und Einrichtung geselliger Zusammenkünfte nach Möglichkeit gethan, was „die Selbstachtung durch äussere Rücksichtnahme zu erhöhen" im Stande ist. Freilich wird das Personal auch durch umfangreichen Wachtdienst, dessen Nothwendigkeit aber ohne Weiteres aus der Schwere des Krankenmaterials erhellt, in Anspruch genommen: auf der Frauen- und Männerabtheilung je sieben Nachtwachen.

M; kommt zum Schluss, dass grössere Anstalten den Vorzug der „Vielseitigkeit der Einrichtungen und genügenden räumlichen- Trennung" haben, "macht aber das Einklassensystem zur Vorbedingung, weniger, weil durch selbstzahlende Kranke der Arzt persönlich stark in Anspruch genommen ist, als weil die Verwaltung verwickelter wird. Verf. befürwortet somit die Trennung der Kranken bemittelter und unbemittelter Stände und verweist erstere in die Privatanstalten; die staatlichen Behörden, die im Gegensatz zu städtischen Gemeinwesen wie Berlin die Fürsorge für Geisteskranke aller Stände übernehmen, können also dieser Forderung nicht gerecht werden, da ökonomische Rücksichten sie zum Bau einer gemeinsamen Anstalt zwingen.

Für Verbrecher wird aus den bekannten Gründen eine eigene Anstalt gefordert.

Hohes Interesse erwecken die Mittheilungen von M.'s Erfahrungen die Behandlung der Alkoholisten und die Familienpflege betreffend. Die Resultate bezüglich des ersten Punktes waren, soweit der Aufenthalt in der Anstalt auch auf den ganz freie Bewegung gestattenden Landhäusern in Betracht kommt, günstige, aber der Heilerfolg versagte bald nach der Entlassung. Die Familienpflege erwies sich für die Alkoholisten weniger geeignet, als für andere Kranke; von diesen seien besonders zwei Kategorien erwähnt:

1. geistig schwächere Personen, die in dem Anstaltseinerlei zu bequem werden und draussen mehr Anregung erhalten,

2. Frauen mit leichten Unruhezuständen, auf die in der Pflege besser Rücksicht von der einzelnen Familie genommen werden kann, während solche Kranke in der Anstalt überall anstossen.

Um die Famienpflege anderwärts einzuführen, ist die Weiterführung der ärztlichen Aufsicht erforderlich, und der Betrieb last sich also überall dort einrichten, wo die Anstalt in oder nahe bei einem grösseren Orte liegt.

Bennecke-Jena.

lieber puerperale Psyehosen. Von 0. Knauer. Berlin 1897. S.

Karger Verf. bespricht die sogenannte Puerperalpsychosen vom Standpunkt Olshausens ', Hansen's nnd Martin Schmidt's. Unter 660 geisteskranken Frauen zählte er 82 Puerperalpsychosen (12,5 pCt.) Von diesen waren 9 zu den Infektionspsychosen, 71 zu den idiopathischen Psychosen und 2 zu den Intoxikationspsychosen im Sinne 0 lshaus en's zu rechnen. Auffällig ist der 
Buchanzeigen.

. geringe Prozentsatz der ersten Gruppe. Ref. findet einen sehr -viel grösseren Prozentsatz. Sämmtliche Krankengeschichten werden, theils kurz theils aus führlicher mitgetheilt. Die beiden Intoxikationspsychosen waren eklamptischen Ursprungs. Belastung war in 54 Fällen nachzuweisen. In 20 Fällen handelte es sich um eine einfache Melancholie. In 40 Fällen war ein de pressives Vorstadium nachzuweisen. Heilung trat in 21 Fällen, Besserung in 35, Tod in 2 Fällen ein. In 24 Fällen wurde Heilung nicht erzielt bezw. erfolgte die Entlassung vor Eintritt der Heilung. Die Arbeit des Verf.'s ist namentlich als statistischer und casuistiseher Beitrag zu der Lehre, von den Puerperalpsychosen in den besseren Ständen, denen seine Kranken durchweg angehörten, zu schätzen.

Diagn ostik der Krankheiten des Nervensystems. Von A. G o 1 d s che id er.

2. vollständig umgearbeitete und wesentlich vermehrte Auflage. Berlin. 1897. Fischer's Med. Buchhandl.

Das Format des bekannten Goldscheider'sehen Buches ist in der neuen Auflage grösser, der Druck übersichtlicher und der Inhalt reichhaltiger geworden. Es ist jedenfalls auf seinem Gebiet weitaus das praktischste und zuverlässigste. Im Interesse künftiger Auflagen möchte Ref. auf einzelne Lücken bezw. Dunkelheiten hinweisen. Im TJntersuchungsschema ist die Eintheilung • der allgemeinen Gehirnfunktionen unrichtig. Auch die parallele Aufführung von „Delirien, maniakalischen Zuständen" entspricht der heutigen Psychopathologie nicht. Moria ist nicht identisch mit abnormer Heiterkeit, vielmehr bezeichnet MoSüu das alberne, läppisch-heitere Wesen mancher Schwachsinnigen. Die Sinnestäuschungen bei Herderkrankungen hätten eine kurze Erwähnung verdient. Die Demenz hat mit der Benommenheit des Sensoriums nichts zu thun. Den Werth des Dynamometers unterschätzt G. Er leistet dem Ref. grosse Dienste, wenn es sich darum handelt, z. B. bei Neurasthenie, die Ermüdung bei öfter in bestimmten Intervallen wiederholtem Druck - zu verfolgen. Die Fehldistanzen bei der Lokalisation von Berührungen sind keineswegs minimal (S. 27). Man findet bei völlig gesunden Individuen Lokalisationsfehler. bis zu $G$ und 7 cm. (Oberarm, Oberschenkel; vgl. die von Lewy bei mir verfasste Arbeit, Zeitschr. f. Phys. u. Psych. 1895). In einer neuen Auflage 'wäre namentlich auch die Wilbrand-sche Methode der Gesichtsfeldaufnahme zu berücksichtigen. Das kalte Bad sollte als Hülfsmittel zur Hervorbringung eines latenten Sehnenphänomens nicht unerwähnt bleiben. Erst vor Kurzem hat sich mir das Mittel wiederum in einem zweifelhaften Falle bewährt. Auch der Scheiber'sche Kunstgriff sollte speziell erwähnt werden. Das Schema S. 49 wird der klinisch doch bedeutungsvollen Thatsache der partiellen Kreuzung der Oculomotoriuswurzel-fasern nicht gerech (vergl. mein Schema im Artikel Pupilleninnervation des Reallexikons der med. Propädeutik). In der Lehre vom Tremor hätte Ref. die Unterscheidung eines Ruhe-Tremor, eines statischen Tremors und eines Intentionstremors gewünscht. Dem zweitgenannten kommt eine ganz bestimmte klinische Stellung zu (vergl. meine Psychiatrie S. $174 \mathrm{ff}$.)

Die topische Diagnostik hat Verf. mit Glück auf die Neuronlehre zu gründen versucht. Die Untersuchungen von Sherrington undHead hätten mehr Berücksichtigung verdient. Die alte Ecker'sche Figur 47 wäre besser durch eine korrektere, z. B. die Eberstaller'sche ersetzt worden. Die Fig. 48 bleibt hinter unseren heutigen Kenntnissen weit zurück.

S. 233 ist das Missverständniss naheliegend, die Tabes entwickele sich erst im weiteren Verlauf I der Dementia paralytica, während viel öfter die Tabes den paralytischen Symptomen vorausgeht. Auch gehört der Grössenund Verkleinerungswahn nicht schon zum Anfangsstadium des Dementia paralytica (S. 232). Die oft so auffällige initiale allgemeine Hypalgesie und die ebenso auffällige Abnahme der groben motorischen Kraft hätten ebenfalls Erwähnung verdient.

Die Alkoholfrage und ihre Bedeutung für Volkswohl und Volksgesundheit. Von August Smith. Eine sozialmedizinische Studie. Tübingen 1895. Oslander'sehe Verlagsbuchhandlung. Die vorliegende Schrift schliesst sich an einen Vortrag an, den Verf.

i. J. 1894 auf der Versammlung südwestdeutscher Neurologen und Irrenärzte 
Buchanzeigen. 
gehalten hat. Sie kann zur Lektüre nur dringend empfohlen werden. Allerdings ist unverkennbar, dass Verf. zuweilen über das Ziel hinausschiesst." Einer vollständigen Umarbeitung müsste in einer neuen Auflage die Lehre von den chronischen Alkoholpsyehosen unterzogen werden. Speziell wird Verf. sich doch entschliessen müssen, die Thatsache anzuerkennen, dass in Folge des AlkoTiolgenusses gelegentlich eine typische chronische halluzinatorische Paranoia vorkommt (vgl. S. 47). Auch die sog. Dipsomanie T>eurtheilt Verf. zu einseitig und gelangt daher auch zu einer viel zu günstigen Prognose (90 pCt. Genesung). Es handelt sich hier um sehr verschiedene Zustände. Ref. unterscheidet folgende:

k Chronische Alkoholisten leichteren Grades, welche in Folge' einer gelegentlichen Verführung (Gesellschaft, Sorge, Aerger) in unregelmässigen Zwischenräumen zunächst zu einem einmaligen stärkeren Excess gelangen und in Folge dieses Excesses tage- und selbst wochenlang in demselben Maasse weitertrinken. Die Veranlassung $\mathrm{zu}$ dem Weitertrinken liegt theils in den gewöhnlichen Katzenjammergefühlen nach dem Excess, theils in der Präcordialangst, welche bei diesem Kranken in Folge eines stärkeren Alkohol-excesses auftritt. Nach dem Anfall kehren diese Kranken zu dem gewohn-heitsmässigen relativ geringeren Alkoholquantum zurück. Diese Fälle sind am häufigsten.

2. Periodische Melancholiker. Die Angst ist primär. Zur Beschwichtigung der Angst trinken diese Kranken excessiv. Die Häufigkeit dieser Gruppe ist von Tuczek entschieden überschätzt worden.

3. Periodische Hypamoniaci. Die Schilderung, welche Verf. S. 44 entwirft, trifft auf diese Gruppe ziemlich gut zu.

Einen viel geringeren Bruchtheil stellt die periodische Paranoia, vereinzelte Fälle auch diaEuilepsie und Hysterie. Strenge, d. Ü. regelmässige Periodizität zeigen übrigens auch die Fälle der 2. und 3. Gruppe selten. Mit der Bezeichnung epileptoid und epileptisch ist Verf. zu freigebig.

Sehr zweifelhaft erscheint. auch die Behauptung des Verfassers (S. 55), dass man den drohenden Ausbruch eines Delirium tremens durch sofortigen Alkoholabbruch und forcierte Ernährung fast ausnahmslos vermeiden könne. Als Beruhigungsmittel wird mit Recht Trional empfohlen.

Die Orientirtheit ist bei dem akuten halluzinatorischen Wahnsinn der Trinker (Paranoia pall. ac.) nicht intakt, wie Verf. S. 57 anzunehmen scheint, sondern nur weniger gestört als bei dem Delirium.

Die Veränderungen der Hirnzellen sind bei dem chronischen Alkoholisten sehr wohl nachweisbar, nicht nur bei eben getödteten Thieren (S. 65).

Mit Recht wendet sich Verf. gegen die Alkohol-Freigebigkeit der Irrenanstalten. Die Schädlichkeit des Tabacks schlägt er erheblich zu niedrig an (S. 67).

Bezüglich der juristischen Behandlung des Trinkers wendet sich Verf. gegen die Entmündigung wegen Trunksucht. Er empfiehlt vielmehr die bekannten Bestimmungen des St. Galler Gesetzes, welches Verf. S. 98 abdruckt.

Prophylaktisch fordert Verf. die allgemeine absolute Abstinenz. Man wird seinen Fragestellungen und Schlüssen immer entgegenhalten können, dass für einen erblich unbelasteten, von neuro- und psychopathischen Symptomen freien, willenskräftigen, über die Folgen des Alkoholismus unterrichteten Erwachsenen die Gefahr eines pathologischen Rauschzustandes oder des Verfallens in chronischen Alkoholismus so gering ist, dass ein leichter Bier- oder Weingenuss unverfänglich ist.

Ref. könnte noch manche einzelne Bedenken gegen diesen oder jenen

Satz erheben. Doch sind diese zu unwichtig gegenüber der Thatsache, dass es dem Verf. im Ganzen sehr gut gelungen ist, dem Arzt und dem gebildeten Laien einen Ueberblick über die Alkoholfrage vom ärztlichen Standpunkt zu geben.

Die Färbeteehnik des Nervensystems. Von B. Pollack.

Berlin. 1897.

S. Karger.

Das kleine Buch des Verfassers genügt einem dringenden Bedurfniss in ausgezeichneter Weise. Es werden ausführlich besprochen:!. Die Technik der Gehirnsection, Methoden zur Conservirung des ganzen Gehirns. Plastische Reproduktion pathol.-anatomischer Präparate. 2. . Härtung, Einbettung, 
Buchanzeigen.

Schneiden. 3. Gewichtsveränderungen des Gehirns in Conservirungsflüssigkeiten. 4. Zeichnen und Photographieren makroskopischer und mikroskopischer Präparate. 5. Färbungsmethoden (S. 43-117).

Nur ganz vereinzelte Lücken finden sich. So vermisst Ref. z. B. die

Lissauer'sche Modifikation der Weigert'schen Methode, welche für $\mathrm{Ge}$

hirne (z. B. Thiergehirne), die zu lange in Chromsalzlösungen und Alkohol

gelegen haben, geradezu unersetzlich ist. Die Nigrosinfärbung fällt meist besser aus, wenn man zunächst in einer Mischung von Wasser und Alkohol zu gleichen Theilen auswäscht.

Atlas des Gehirns. Herausgegeben von Prof. Dr. Carl Wernicke. Abtheilung 1, 32 Frontalschnitte durch eine Grosshirnhemisphäre. Her- j gestellt und erläutert von Dr. Ernst Hahn und Dr. Heinrich Sachs. Commissionsverlag der Schlötter'sehen Buchhandlung, Breslau 1896. Ueber den Nutzen der Photographie für die bildliche Darstellung von Schnittpräparaten oder ähnlichen Dingen sind die Meinungen noch sehr ge-theilt. Niemand wird freilich leugnen, dass die Photographie gegenüber den Handzeichnungen gewisse Vortheile hat. Vor allem giebt sie ja die Bilder ohne jede subjective Zuthat des Beobachters, so dass jeder Leser einer Abhandlung sich über die Deutung der dargestellten Dinge ein Urtheil bilden kann, ohne dass er gezwungen wäre, selbständige Nachuntersuchungen anzustellen. Dieser Vortheil kommt besonders dann zur Geltung, wenn es sich einmal um sehr dünne Präparate handelt, und dann um solche, bei denen nur ein kleiner Theil des Object's zur Betrachtung gelangen soll. Als ganz besonders fruchtbringend hat sich daher die photographische Re-production bei den Bacterien erwiesen, freilich auch nur dann, wenn es sich bei diesen nicht um Schnittpräparate, sondern um Anstriehobjecte handelte. In solchen kann man ja die Bacterien in ganz ausserordentlich dünner Schicht und ohne störende andere mikroskopische Beigaben ausbreiten. Bei Bacterien kommt es auch nicht darauf an, einen grossen Theil des Objects zur scharfen Abbildung zu bringen, es genügt, wenn nur einige Exemplare • gerade der in Frage kgmmenden Bacterien im Gesichtsfelde ganz deutlich wiedergegeben Werden. ' Anders liegt die Sache bei Schnittpäparaten, selbst von Bacterien, erst recht aber bei andern Objecten. Hier und da gelingt es ja einem besonders geübten mikroskopischen Photographen, recht brauchbare Abbildungen zu Stande zu bringen, aber im allgemeinen sieht nur der etwas an den mikroskopischen Photographien, der die Präparate selbst kennt. Gerade der aber täuscht sich gewöhnlich ausserordentlich über die Klarheit seiner Photographien für fremde Leser. Eine Anzahl vortrefflicher Abhandlungen ist wegen der photographischen Abbildungen mikroskopischer Präparate ungemein schwer zu verstehen. Dem Schreiber ist eine mittelmässige Handzeichnung mikroskopischer Objecte immer noch viel lieber, als derartige Photographien. Es ist ja richtig, dass die Zeichnung stets etwas subjectives enthält, aber man sieht doch" wenigstens, was' der Verfasser gemeint hat, während man photographischen Reproductionen ver-grösserter Objecte oft rathlos gegenübersteht.

Ganz anders liegt die Sache, wenn es sich darum handelt, makroskopische Objecte zur Darstellung zu bringen, oder mikroskopische Schnitte ohne Vergrösserung abzubilden. Man betrachte nur die Abbildungen makroskopischer Gehirntheile in dem prachtvollen Atlas von Gustav Retzius. Dieser Atlas bringt nur anatomisch präparirte Gehirntheile, jetzt aber ist gewissermassen eine Ergänzung dieses Atlasses erschienen, der unter Re-daction von Wernicke von dessen Assistenten Hahn und Sachs auf photographischem "Wege hergestellt ist. Dr. Sachs hat die Schnitte angefertigt, Dr. Hahn hat dieselben photographirt. Die Schnitte sind ohne Vergrösserung wiedergegeben.

' Dass Schnitte in ihrer natürlichen Grösse sich viel leichter scharf abbilden lassen, als vergrösserte, liegt auf der Hand. Namentlich kommt dabei in Betracht, dass-bei mikroskopischen Vergrösserungen ja nicht nur die Fläche des Schnittes vergrössert wird, sondern auch seine Dicke. Je weiter nun die obere und die untere Fläche eines Schnittes im mikroskopischen Bilde von einander abweichen, um so schwerer wird die scharfe Einstellung sich bewerkstelligen lassen, um so mehr werden sich 
die nicht scharf eingestellten Theile des Präparates als störende Schatten über die scharf eingestellten herüberlegen und das Bild unklar machen. Bildet man die Schnitte aber ohne Vergrösserung ab, so entspricht die Dicke des Bildes auch beim Photographiren der naturlichen Schnittdicke, und der Fehler wird wesentlich geringer-, als bei der Abnahme vergrösserter Prä-arate. Auch hierbei werden die Schnitte ja wohl auch kaum mit der chärfe einer Handzeichnung wiederzugeben sein, aber man nimmt diese geringere Schärfe gern mit in den Kauf, wenn es sich darum handelt, alles Subjective aus den Bildern auszuschalten. "Wie nöthig das gerade für die gröberen Verhältnisse des Gehirns ist, ergiebt sich aus der Vergleichung älterer Abbildungen des Gehirns mit den heutigen, oder mit den Photographien des Retzius'sehen Atlas. Gerade weil am Gehirn noch so •vieles in seiner Bedeutung unbekannt ist, muss bei bildlichen Darstellungen desselben, die einen bleibenden "Wert beanspruchen sollen,. das Subjective möglichst fern gehalten werden. Freilich'so einfach, wie bei den Bildern des genannten Atlasses von Retzius, lag die Sache bei der Aufgabe, die sich Wernicke und seine Mitarbeiter gestellt hatten, durchaus nicht.

Bei den grob anatomischen Bildern genügt der natürliche Helligkeitsunterschied an den verschiedenen Theilen des. Objectes vollkommen, um ein scharfes photographisches Bild zu.Stande zu bringen, bei Schnittpräparaten, selbst wenn dieselben nicht vergrössert werden sollen, ist das aber nicht der Fall. Hier muss die künstliche Färbung des Objects dazukommen, wenn man deutliche Bilder haben will. Färbungen für die Gehirnbestand-theile haben wir ja, aber das, was bei der Betrachtung der Schnitte mit blossem Auge eine genügende Differenzirung abgab, war noch lange nicht für die photographische Wiedergabe recht geeignet. Die Schnitte mussten daher überdifferenzirt werden. JDas wäre für die wirklich mikroskopische Betrachtung der Präparate freilich sehr wenig vortheilhaft gewesen. Für die rein makroskopischen Bilder kam das nicht in Betracht, aber ein Zuviel musste auch hierbei vermieden werden.

Diese Schwierigkeit war verhältnissmässig leicht zu überwinden. Grösser war schon eine andere, die durch die erforderliche Grösse der Schnitte bedingt war. Die Schnitte gingen ja durch eine ganze Hemisphäre des menschlichen Gehirns, und solche Schnitte sind recht schwer gut anzufertigen und zu manipuliren. Auch die rein photographische Technik stiess auf grosse Schwierigkeiten, die erst durch eine unendliche Anzahl von Experimenten so weit wie möglich überwunden wurden und dergl. mehr.

Wir müssen daher den Herren Hahn und Sachs, die sich um die Herstellung der Photographien so sehr bemüht haben, sehr dankbar sein. Sie haben es aber auch zu Stande gebracht, dass der Atlas nunmehr eine Art „Standardwerk" darstellt. Man kann an den Abbildungen einmal den normalen "Bau des Grosshirns studiren, soweit derselbe an solchen Schnitten, die ja nicht jeder sich anfertigen kann, makroskopisch zu erkennen ist, und man kann etwaige abnorme Präparate dieser Art mit den normalen Abbildungen des Atlasses vergleichen. Es ist schon eine grosse Bequemlichkeit für die Darstellung, wenn man sagen kann: „Auf Schnitt 57 des Wernicke'schen Werkes war das und das verändert."

Freilich wäre das Werk doch recht unvollständig gewesen, wenn es nicht der blossen so zu sagen mechanischen Wiedergabe der Hirnschnitte auch ein geistiges Moment hinzugefügt hätte. Dieses besteht einmal in der jeder Tafel beigegebenen schematischen Pause, sodann in dem dem Atlas beigefügten erläuternden Texte, in welchem die Bilder eingehend besprochen sind.

Bis jetzt sind als Abtheilung 1 des Werkes 32 Frontalschnitte durch eine Grosshirnsphäre erschienen, deren Entfernung von dem Stirnpol des Gehirns in Millimetern angegeben ist.

Die Herstellung des Werkes, das ja beträchtliche Geldopfer erforderte, ist durch die Munificenz der Königlich prenssischen Akademie der Wissenschaften ermöglicht worden.

Zum Schlüsse will ich nicht unterlassen, darauf aufmerksam zu machen, dass Probetafeln auf Wunsch von der Verlagsbuchhandlung zur Ansicht geliefert werden.

Frankfurt a. M. 


\section{Tagesgeschiehtliche Notizen.}

In der Nummer des Progres medical vom 9. Mai dieses Jahres äussert Bourneville Bedenken gegen die Abhaltung eines zweiten internationalen Congresses in Brüssel. Man wird in der Tnat nicht leugnen können, dass die Congresshäufung in diesem Jahre ein Maximum erreichen wird.

In Philadelphia starb Hugo Engel, Professor der Neuropathologie; in Tokio Sakaki, Professor der Psychiatrie und Neurologie.

Bekanntlich ist noch strittig, in welchem Umfang ungefährliche an Dementia senilis leidende Kranke in Irrenanstalten unterzubringen sind. Der Präfekt des Seinedepartements hat neuerdings durch einen Erlass die Ueber-führung solcher Kranken aus den gewöhnlichen Spitälern in die Irrenanstalten des Departements auf Fälle unmittelbarer Gefahr zu beschränken befohlen. Da auch bei uns eine ähnliche Frage binnen kurzem zu erörtern wird, verzeichnen wir diesen Versuch an dieser Stelle, um später über das Ergebniss zu berichten.

Die 23. Versammlung der American Neurological Association hat am 4.-6.Mai in Washington stattgefunden. Wir werden einen ausführlichen Bericht bringen, ebenso auch über die Versammlung der American Medico-psycho-logical Association in Baltimore am 11.-14. Mai.

Die Societe medicopsychologique hat am 3. Mai die Arbeiten von Battier und Lelong „Essai sur hallucinations motrices verbales", von R i e d e r „Recherches sur les urines ä la deuxieme periode de la paralysie generale", und von Bonnet und von Maupate „Du langage chez les idiots" mit Preisen gekrönt.

Der zweite Congres national d'assistance wird in Rouen am 14. Juni abgehalten. Auf der Tagesordnung steht $u$. A. die Frage der Unterbringung der Epileptiker und der Fürsorge für entlassene Geisteskranke.

Die 65. Versammlung der British Medical Association findet in diesem Jahr in Montreal statt. Der Beginn ist auf den 31. August festgesetzt. Chairman der psychiatrischen Section ist Dr. R. M. Buche.

\section{Berichtigung.}

In dem vorigen Heft (No. 5) sind in dem Aufsatz des Dr. Mann „Ueber das Verhalten der Sehnenreflexe und der passiven Beweglichkeit bei der Hemiplegie" die Seiten No. 425 und 426 versehentlich beim Druck mit einander vertauscht worden. Die betreffende-Stelle ist also in der Reihenfolge:

Seite 424, 426, 425, 427 -zu lesen.

Verantwortlicher Redakteur: Prof. Dr. Th. Ziehen in Jena. 